\title{
Brésil : panorama général du projet de la première ligne à grande vitesse
}

Project overview: The first high-speed railway line in Brazil

Brasilien : Übersicht des Erstes Hoch Geschwindigkeit Projekts

\section{Francisco Cortezzi}

\section{OpenEdition}

\section{Journals}

Electronic version

URL: https://journals.openedition.org/rge/5643

DOI: $10.4000 /$ rge. 5643

ISSN: 2108-6478

\section{Publisher}

Association des géographes de l'Est

Printed version

Date of publication: 1 October 2015

ISSN: 0035-3213

\section{Electronic reference}

Francisco Cortezzi, "Brésil : panorama général du projet de la première ligne à grande vitesse", Revue Géographique de l'Est [Online], vol.55 / n³-4 | 2015, Online since 17 October 2015, connection on 21 September 2021. URL: http://journals.openedition.org/rge/5643 ; DOI: https://doi.org/10.4000/rge. 5643

This text was automatically generated on 21 September 2021.

Tous droits réservés 


\title{
Brésil : panorama général du projet de la première ligne à grande vitesse
}

\author{
Project overview: The first high-speed railway line in Brazil \\ Brasilien : Übersicht des Erstes Hoch Geschwindigkeit Projekts
}

Francisco Cortezzi

\section{Introduction générale}

1 La vitesse est sans doute une déterminante essentielle de nos comportements spatiaux et territoriaux. Selon BAVOUX et al. (2011), « la vitesse appelle la vitesse». Toujours selon ces auteurs, la vitesse entraîne aussi des améliorations de la qualité de la vie pour la frange la plus aisée de la population. On peut ainsi évoquer une dromocratie, c'est-àdire une société où les plus riches sont aussi les plus rapides dans leurs mobilités (BEYER, 2014). Pour les entreprises, les gains de temps peuvent susciter de nouvelles dynamiques sinon des effets structurants : le client peut aller plus loin si cela en vaut la peine, et les services peuvent s'installer si la clientèle a la capacité de venir de loin.

2 A ce jour, le Brésil est un pays d'avions, de voitures et de camions. Mais en 2007, désireux de rejoindre le contexte de la grande vitesse ferroviaire, le gouvernement brésilien a proposé le projet de la première Ligne à Grande Vitesse (LGV). Ce projet consiste à parcourir $511 \mathrm{~km}$ en connectant trois villes de la région Sud-Est du Brésil : Rio de Janeiro, São Paulo et Campinas.

3 Du fait de la pénurie d'informations solides et substantielles, le support de notre travail repose sur le projet élaboré, en 2009, par le Consortium Halcrow-Sinergia. En 2008, la Banque Interaméricaine de Développement (BID) a commandité ce consortium afin d'évaluer la viabilité technique et économique/financière de la ligne à grande vitesse Rio de Janeiro - São Paulo - Campinas. Cet projet est disponible sur le site internet http://www.antt.gov.br/index.php/content/view/13208/DEMANDA_E_RECEITA_.html. 
4 Avant d'en venir au projet lui-même, nous présenterons, de manière générale, la région concernée par son implantation.

\section{A. La région Sud-Est}

Le Brésil est subdivisé en 5 grandes régions administratives (Figure 1) : Nord, Nord-Est (généralement écrit à la brésilienne, Nordeste), Sud-Est (Sudeste), Sud et Centre-Ouest.

Figure 1 : Brésil, division par États et régions

\section{Brésil: division par États et Régions}

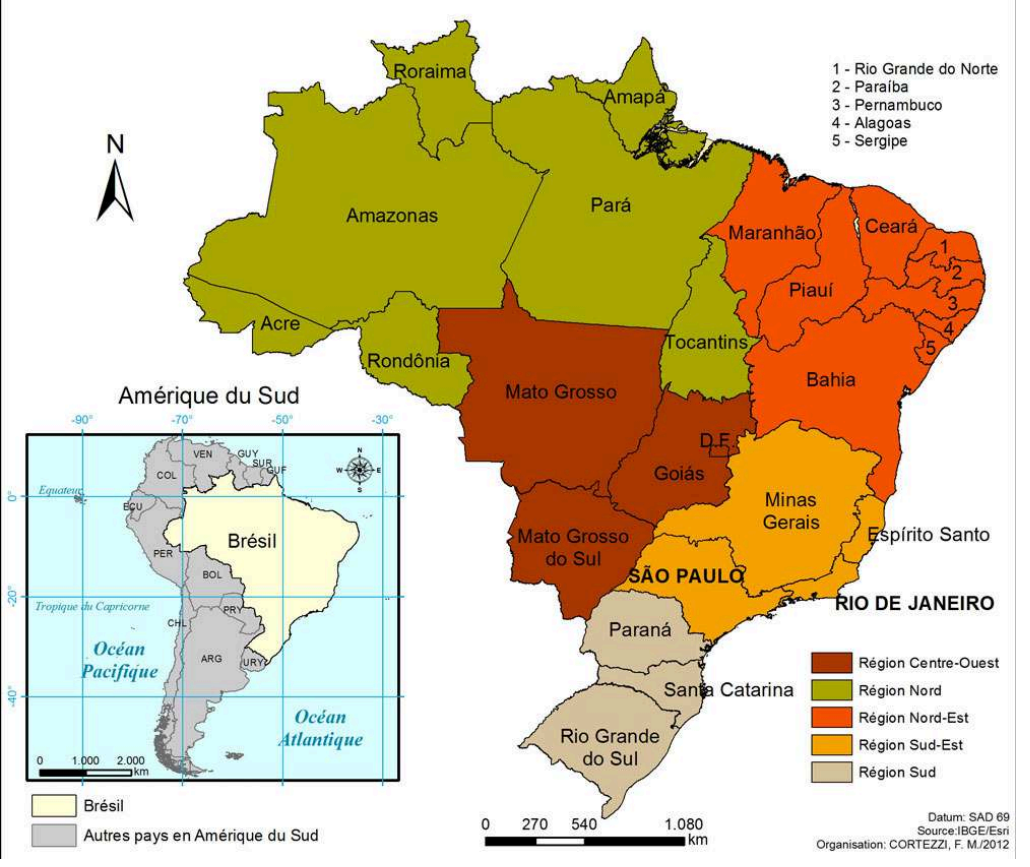

Source : IBGE

6 En rassemblant $10 \%$ du territoire brésilien, $80 \%$ de la population totale et $55 \%$ du PIB, la région Sud-Est correspond a priori le mieux aux caractéristiques attendues pour le développement de la grande vitesse ferroviaire. En effet, par sa géographie et son économie, la région Sud-Est se détache des autres territoires. Hervé Théry (2012) fait une remarque intéressante à ce sujet :

«On a dit du Brésil, pour illustrer de façon frappantes ses disparités régionales, qu'il évoquait « une Suisse dans un Pakistan ». Il y aurait à réduire sur le choix des pays, car si le Pakistan n'illustre pas trop mal le degré de développement de ses régions pauvres, le style de croissance du Sudeste évoque assez peu celui de la Confédération helvétique : peut-être le nom créé par Edmar Bacha, Belindia, formé en associant les noms de la Belgique et de l'Inde, serait-il plus approprié » (THÉRY, 2012, p. 77).

7 La Figure 2 confirme ce commentaire à propos des grandes disparités régionales du Brésil. Dans son entier, le Brésil compte 27 Régions Métropolitaines formant des ensembles de communes indépendantes fondées sur une communauté socioéconomique d'intérêts et collaborant en principe dans ce but. L'État de São Paulo compte trois de ces régions: depuis 1973 la Região Metropolitana de São Paulo 
(19.092.978 habitants en septembre 2005) ; au nord, à 120 km, la Região Metropolitana de Campinas (2.631.241 habitants) ; au sud, depuis 1992 la Região Metropolitana de la Baixada Santista autour du port de Santos avec 9 municipalités en ruban sur $125 \mathrm{~km}$ le long du littoral (1.639.641 habitants). Ces régions métropolitaines peuvent à leur tour créer un échelon propre. Ainsi, le Grand São Paulo Concerne 39 communes établies sur $8.051 \mathrm{~km}^{2}$ dont $25 \%$ sont urbanisés. Il est intéressant de relever que lors du passage à l'an 2000, le slogan de cette entité était « il est temps de s'organiser », ce qui témoigne d'une prise de conscience en matière de planification régionale.

Figure 2 : Brésil, Vue de nuit de l'espace

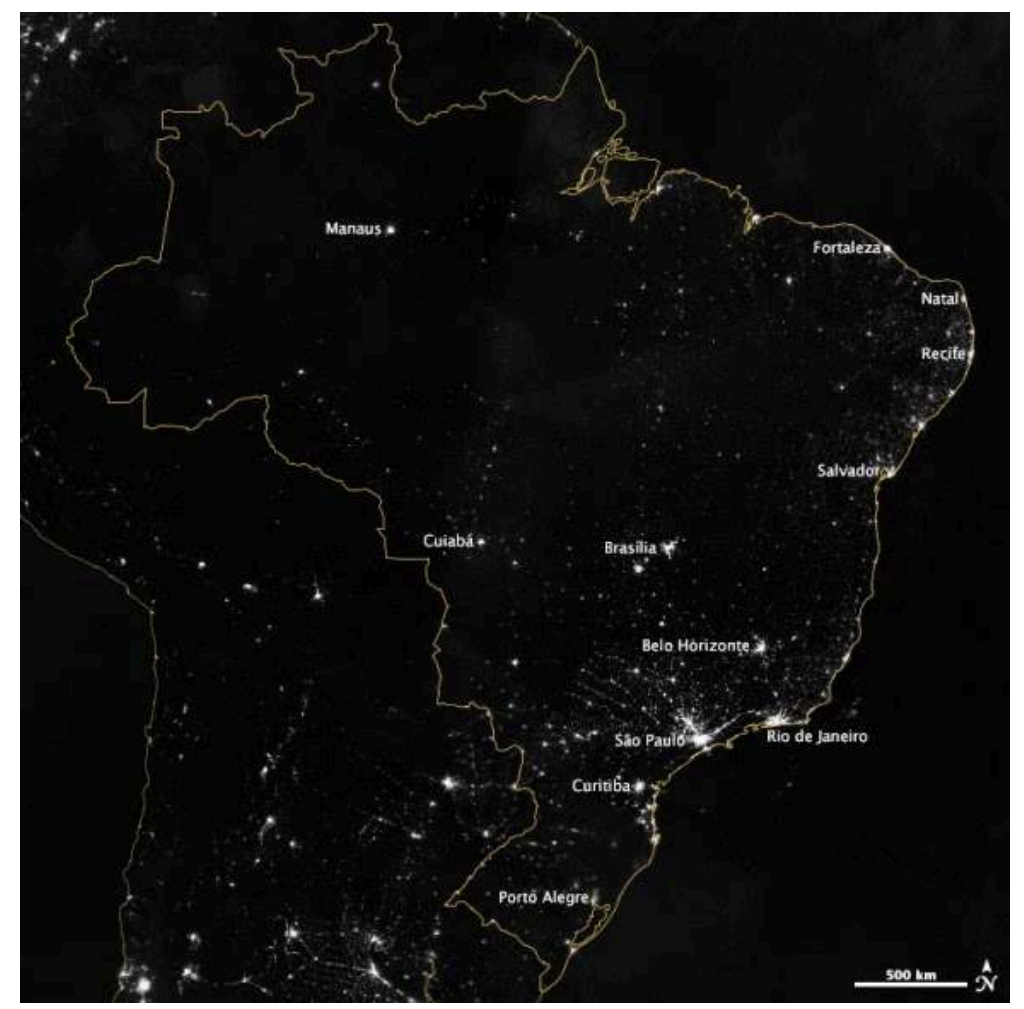

Source : http://pt.wikibooks.org/wiki/Urbaniza\%C3\%A7\%C3\%A30/Malhas_urbanas

\section{B. São Paulo, Rio de Janeiro et Campinas}

8 La région Sud-Est se subdivise en 4 États : São Paulo, Rio de Janeiro, Minas Gerais et Vitória. De la même manière que la région Sud-Est se distingue au sein des autres régions du Brésil, les États de São Paulo et Rio de Janeiro se différencient du Minas Gerais et de Vitória. La somme de la population des deux États est de 57.252.128 habitats, alors que le total de la région Sud-Est est de 80.364.410 habitants (IBGE, 2010). Les villes de São Paulo, Rio de Janeiro et Campinas sont les principales agglomérations du pays ; elles voient se développer des processus de conurbation (Figure 3). 


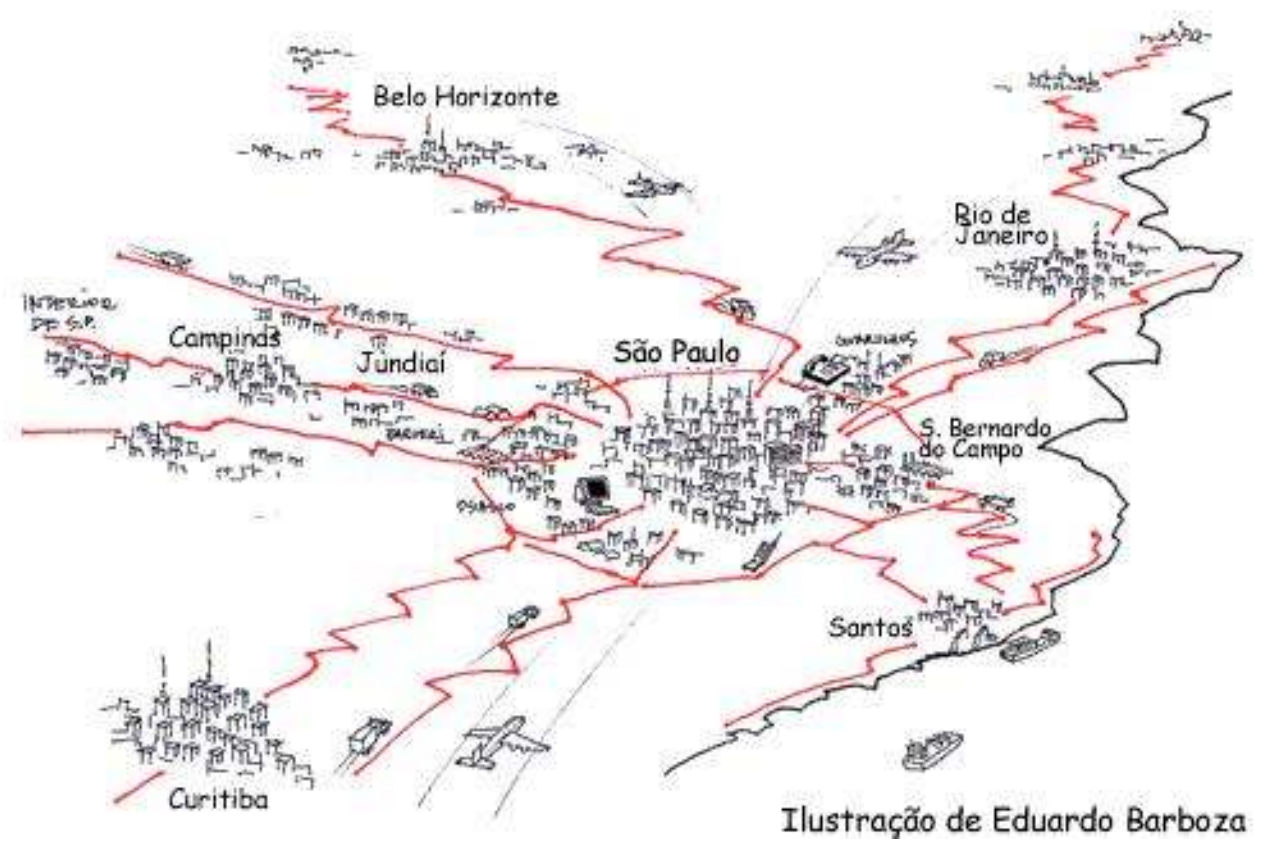

Source : Architectura Moderna, http://arquiteturamoderna.blogspot.fr/

9 La Figure 3 met en évidence la grande concentration de la population dans la portion méridionale de la région Sud-Est. Le corridor Rio-São Paulo apparaît dans sa densité, mais l'ensemble São Paulo-Campinas affirme un processus d'urbanisation bien plus intense. Selon une disposition radiale, São Paulo satellise toute une série d'agglomérations dans son aire d'expansion. Conformément au slogan de 1954, lors du 400 e anniversaire de sa fondation, on a bien là « la ville qui ne s'arrête jamais ». D'un point de vue symbolique, la grande vitesse apparaît logique dans ce contexte de villes entreprenantes et fières de s'afficher.

\section{La ville de São Paulo}

10 La ville de São Paulo est le centre économique, commercial et industriel du Brésil. Toujours selon encore Théry (2012), «le centre de São Paulo évoque Tokyo ou Manhattan ». Avec une population de 11.259.503 habitats (IBGE, 2010), la ville possède des relations aériennes directes avec plus de 25 pays. Elle dispose également du meilleur et plus grand système de métro du Brésil, avec 78,2 kilomètres de lignes. Sa zone métropolitaine compte plus de 19 millions d'habitants et l'une des villes les plus importantes dans ce conglomérat urbain est Campinas.

\section{La ville de Campinas}

11 Avec une population de 1.080.113 habitats (IBGE, 2010), la ville de Campinas est un pôle industriel très important, en particulier dans le secteur automobile. Honda, Toyota, Bosch, 3M, Bridgestone, Pirelli sont quelques-unes des grandes entreprises opérant dans la ville. Grâce à cette base industrielle, Campinas est désormais entrée dans une stratégie post-industrielle avec un important secteur de production scientifique. Elle 
est en outre bien placée dans le contexte aérien national. Tous ces attributs font de Campinas un centre secondaire important dans la région Sud-Est.

\section{La ville de Rio de Janeiro}

Pôle touristique et événementiel majeur du Brésil sinon du monde (Coupe du monde de football en 2014, Jeux olympiques en 2016), la ville de Rio de Janeiro est considérée comme une des "plus belles villes au monde ». Avec 6320446 habitats (IBGE, 2010), la " cidade maravilhosa » possède des relations très fortes avec l'ensemble des villes au Brésil et, principalement, à cause du tourisme, avec le monde entier.

\section{Le projet « TAV » Brésil}

Le projet TGV Brésil (en portugais, TAV - Train à Haute Vitesse) a pour but de réaliser la liaison entre les villes de Rio de Janeiro et Campinas via São Paulo. La longueur totale est de $511 \mathrm{~km}$ avec un voyage estimée d'environ 2 heures et 25 minutes (en fonction du trajet préliminaire et des arrêts intermédiaires prévus), tandis qu'un service direct entre São Paulo et Rio de Janeiro serait d'environ 1 heure et 33 minutes, et un peu plus dans la direction opposée.

14 Le projet est basé sur la technologie générique des trains à grande vitesse, avec une estimation spécifique pour un tracé exclusif, de façon à maximiser la vitesse de déplacement et à assurer une performance opérationnelle élevée en termes de fiabilité et de ponctualité. D'emblée, il faut relever la différence d'altitude entre les deux villes qui génère une complication technique. Si Rio de Janeiro est au niveau de la mer, São Paulo se trouve sur un plateau à environ 800 mètres de haut.

15 Il est prévu que le TAV sera un mélange de nouvelles stations et stations revitalisés. Il y a des plans pour revitaliser et améliorer les stations abandonnées comme à Rio de Janeiro et à Campinas. Pris dans son ensemble, le projet initial prévoit des gares dans les villes de São José dos Campos (629.921 hab.), Aparecida do Norte (35.007 hab.), Resende (119.769 hab.) et Volta Redonda (257.803 hab.). Les aéroports de Viracopos (Campinas), Guarulhos (São Paulo) et Galeão (Rio de Janeiro) devraient également être connectés à la LGV (Figure 4). 
Figure 4 : Le projet LGV Brésil

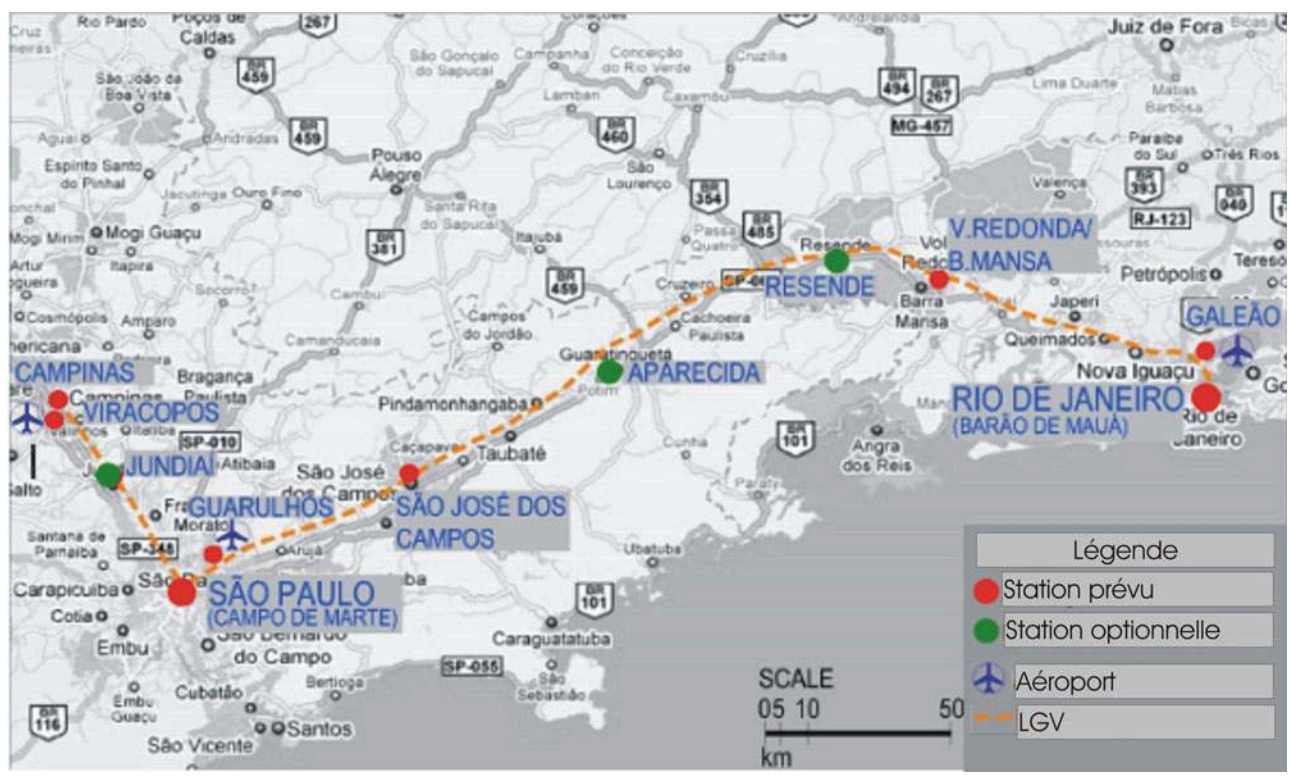

Source : Projeto TAV/Relatório Final, 2009 entre São Paulo et Rio de Janeiro est d'environ $412 \mathrm{~km}$. Basé sur le trajet préliminaire développé (Figure 4), le temps de déplacement, sans arrêts, entre ces dernières deux villes est estimé à environ 1 heure 33 minutes. Les temps des parcours pourront varier en fonction du nombre d'arrêts, avec une grande vitesse de service de longue distance entre Rio de Janeiro et Campinas, en prenant jusqu'à 2 heures et 27 minutes (les temps de parcours sont approximatifs et sont basés sur une vitesse d'exploitation maximale de 300 kilomètres par heure). On aurait donc des TGV bolides et des TGV maillant plus finement les dessertes, à l'image de ce qu' l'on peut observer sur le réseau japonais à grande vitesse.

Le TGV-Brésil mêlera de nouvelles gares et des gares revitalisées. À Rio de Janeiro, il y a des plans pour revitaliser la gare abandonnée Barão de Mauá $(\mathrm{km} \mathrm{0})$ qui se trouve à côté de la principale gare routière Novo Rio. On prévoit en outre un centre technique pour 
la maintenance au même endroit. La gare suivante consiste en une nouvelle station souterraine qui desservira l'aéroport international de Rio de Janeiro - Galeão - (km 15). À partir du Galeão, la ligne monte vers la Serra do Mar, ce qui constitue un des défis techniques en nécessitant de nombreuses sections de tunnels et de viaducs. Une autre station est prévue à de Volta Redonda, toujours dans l'État de Rio de Janeiro (km 118).

Vers l'ouest, le TGV traverserait la limite des États de Rio de Janeiro et de São Paulo. Une station est prévue à la ville d'Aparecida. Cette ville est un important centre de pèlerinage qui a reçu 9,5 millions de visiteurs en 2008 (Secrétaire du Tourisme Brésilien). Après Aparecida, le TGV atteint alors la grande ville industrielle de São José dos Campos ( $k m$ 327). São José dos Campos est un important centre de haute technologie aérospatiale. La ville abrite l'usine d'assemblage d'Embraer, l'entreprise brésilienne d'aéronautique. On se propose d'y construire un deuxième centre de maintenance pour le matériel roulant.

21 Un peu plus vers l'ouest, à la sortie de São José dos Campos, une gare est prévue dans l'aéroport international de São Paulo - Guarulhos - (km 390). La gare de l'aéroport de Guarulhos sera souterraine, à proximité des principaux bâtiments du terminal. En arrivant à São Paulo, l'endroit a priori préféré pour la station est le Campo de Marte ( $\mathrm{km}$ 412) qui est actuellement un aérodrome fédéral situé dans la région nord de la ville. Selon l'avis du Consortium Halcrow-Sinergia, le choix du Campo de Marte offre possibilité de construire une gare d'envergure, au-delà de l'avantage apporté par de sa bonne localisation.

22 A partir de São Paulo, le parcours du TGV s'infléchirait au nord-ouest, vers la ville de Campinas. Au nord de São Paulo, il y a des conditions intéressantes pour la construction d'une autre gare, à ce jour optionnelle, dans la ville de Jundiaí, située entre les autoroutes Anhaguera et Bandeirantes. Le trajet va ensuite vers le nord, jusqu'à

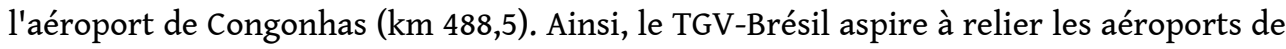
Guarulhos, Galeão et Congonhas aux grands centres urbains. Enfin, la dernière gare serait celle de Campinas ( $\mathrm{km} \mathrm{511).}$

\section{B. Les modes de transport dans la région}

Comme nous l'avons évoqué au début, le Brésil est un pays d'avions, de voitures et de camions. En ce qui concerne le transport de voyageurs, ces modes sont bien établis sur le marché entre les trois villes (Rio de Janeiro, São Paulo et Campinas). En particulier, il faut souligner l'importance du « skybridge » qui relie les villes de Rio de Janeiro et São Paulo. En 2008, la demande entre ces deux villes a été de 7,3 millions de voyages, avec une part du marché de $60 \%$ pour le transport aérien, $17 \%$ pour le transport en voiture et $23 \%$ pour le transport par bus (Figure 5 ). 
Figure 5 : Modales et motifs de déplacement entre Rio de Janeiro et São Paulo

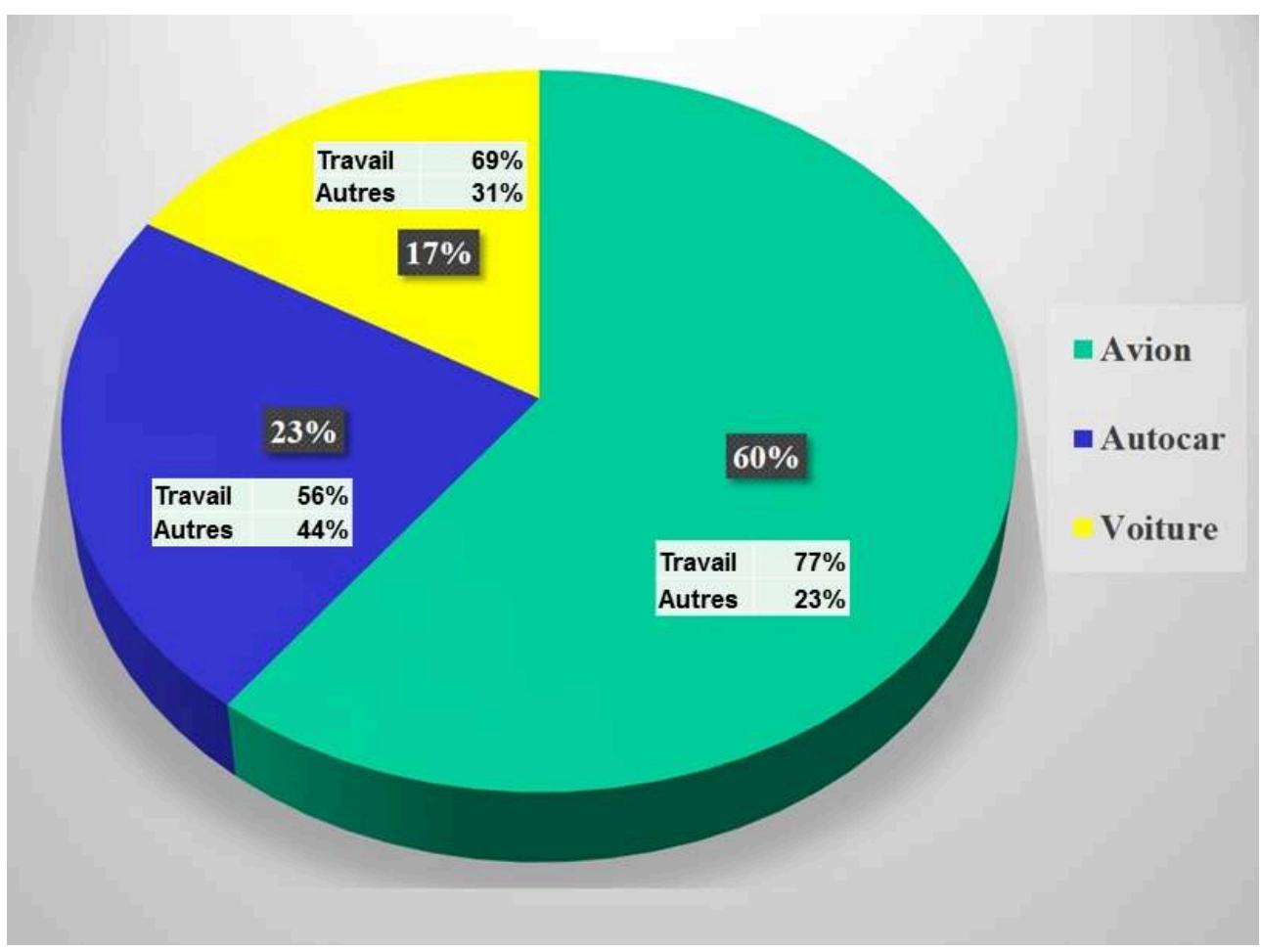

Source : Projeto TAV/Relatório Final, 2009 minutes entre la porte d'embarquement et la porte de débarquement, offre actuellement 71 vols quotidiens dans chaque sens, et domine à l'évidence le marché des passagers d'affaires sensibles au facteur temps. Les deux aéroports nationaux, Santos Dumont (Rio de Janeiro) et Congonhas (São Paulo), sont exceptionnellement bien situés pour les marchés qu'ils desservent, car ils se trouvent à proximité des zones commerçantes et des quartiers d'affaires des centres de Rio de Janeiro et de São Paulo. Les aéroports internationaux (Galeão à Rio de Janeiro et Guarulhos à São Paulo), en revanche, sont situés à 27 et $20 \mathrm{~km}$ respectivement des centres commerciaux, en nécessitant, en taxi, un temps de parcours compris entre 30 et 60 minutes. Ainsi, l'accessibilité des aéroports nationaux contraste avec les projets de trains à grande vitesse dans d'autres pays, dans lesquels les aéroports concurrents sont généralement situés à la périphérie, tandis que les gares sont situées dans le centre-ville.

Toutefois, les aéroports nationaux de Congonhas et Santos Dumont souffrent de congestion. Un grave accident en 2007, à Congonhas, dans des conditions météorologiques défavorables, a mis en évidence les problèmes de l'exploitation d'un service de haute intensité. Congonhas fonctionne actuellement avec un excédent de capacité, et pour essayer de maximiser la capacité disponible, l'aéroport de Santos Dumont est presque entièrement dédié au service à São Paulo. En raison de leur emplacement respectif, l'expansion de l'un ou des deux aéroports serait difficile et coûteuse. Toutefois, le «skybridge » est une opération extrêmement efficace qui offre des services fréquents et situés à proximité des zones commerciales centrales des deux villes. 


\begin{abstract}
Janeiro, São Paulo et Campinas sont desservies par un réseau de routes à péage, de bonne qualité. Les services de bus sont bien utilisés et offrent trois niveaux de service. Rappelons qu'il n'existe plus de train de passagers entre les trois villes.
\end{abstract}

Les trajets en voiture et bus entre Rio de Janeiro et São Paulo prennent entre 5 et 6
heures. Ils subissent des embouteillages dans les zones urbaines. Les villes de Rio de

\title{
III. Les principales aspirations
}

Outre l'importance des éléments déjà cités, quatre objectifs à remplir par le TAV sont fortement mis en évidence :

- la diminution du trafic pour l'autoroute « Presidente Dutra », principale liaison terrestre entre Rio de Janeiro et São Paulo (environ 90000 voyages/jour) ;

- le désengorgement des aéroports « Santos Dumont ", à Rio de Janeiro et « Congonhas ", à São Paulo (71 vols/jour dans chaque sens entre ces deux villes) ;

- l'importation de nouvelles technologies ;

- la réduction des émissions de $\mathrm{CO} 2$.

\section{Des difficultés insurmontables ?}

Parmi les diverses difficultés qu'un projet de cette dimension englobe, deux d'entre elles sont principalement mises en évidence : le milieu physique, qui nécessite la construction de nombreux ouvrages d'art, et les problèmes d'expropriation.

En ce qui concerne la topographie, le principal aspect à prendre en compte est le gradient altimétrique entre Rio de Janeiro et São Paulo. La première ville se trouve au niveau de la mer et São Paulo se situe à environ 800 mètres d'altitude. Tout au long du parcours, la Serra do Mar (une chaîne de montagnes qui s'étend sur environ $1.500 \mathrm{~km}$ le long de la côte sud-orientale allant de l'Etat de Rio de Janeiro jusqu'au nord de l'Etat de Santa Catarina) constitue un obstacle considérable dont il faut pouvoir s'affranchir. Il est donc nécessaire de construire des ponts et des tunnels qui représentent environ $39 \%$ du total du trajet et $52 \%$ du coût total du projet (Figure 6). On se retrouve donc dans un contexte plus japonais qu'américain ; en Californie, le projet de ligne à grande vitesse a la chance de pouvoir utiliser des plaines entre San Francisco et Los Angeles. 
Figure 6 : Croquis schématique des ponts et tunnels

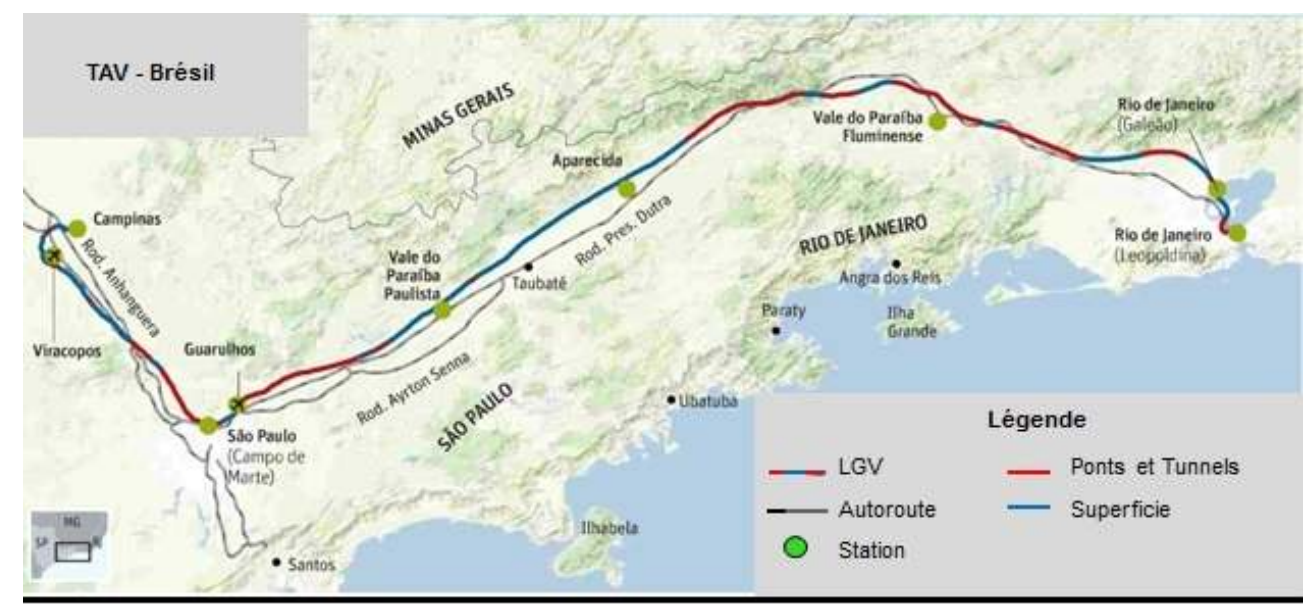

Source : http://www.skyscrapercity.com/showthread.php?t=395459\&page=481

La densité de la population de la région Sud-Est et, surtout, l'intensification du processus de conurbation entre les trois villes viennent compliquer la situation. Il sera nécessaire réaliser des expropriations dont le coût est estimé à 1,3 milliard d'euros, ce qui correspond à $12 \%$ du total du budget du projet.

\section{Remarques finales}

Pour la première année, les études estiment que le TAV transporterait environ 6,4 millions de voyageurs entre Rio de Janeiro et São Paulo et 12,4 millions entre São Paulo et Campinas (Projeto TAV/Relatório Final, 2009). Selon encore les études préliminaires, les gares les plus importantes seraient celles de São Paulo et de Campinas, avec, respectivement, environ 75.000 voyageurs/jour et environ 40000 voyageurs/jour. Autrement dit, la plus grande part du trafic correspondrait à un barreau court qui renforcerait sans doute un effet-banlieue pour Campinas, alors que la liaison intermétropolitaine serait secondaire. C'est ainsi que la répartition des coûts de construction et l'exploitation du TAV montre un autre fait paradoxal : les deux tiers du coût total du projet concernent le tronçon qui présente la demande la plus faible (Junqueira, 2012).

Dans ce panorama général, il est également important de souligner que depuis 2007, quatre tentatives de réalisation ont échoué les unes après les autres. La première avait été prévue pour 2014, avec l'objectif de répondre aux besoins de la Coupe du Monde de Football (où les matchs plus importants ont eu lieu dans la région Sud-Est). La deuxième était prévue pour 2016 avec l'objectif de favoriser les mobilités lors des Jeux Olympiques à Rio de Janeiro, la troisième pour 2017 et la dernière pour 2020. Mais tout au long de ce parcours, il y a eu deux annulations d'appels d'offre. En 2010, il n'y avait pas eu d'entreprises intéressées. En 2013, seule une entreprise française (Alstom) avait répondu à l'appel d'offres, mais les entreprises allemande et espagnole ont demandé quant à elles une nouvelle date limite pour leur soumission au projet.

Somme toute, il est possible de se demander quel est le vrai objectif du TAV. Lorsque l'on songe aux retards et aux manques d'investissements dans le transport ferroviaire brésilien, il apparaît surprenant de passer directement à la technologie à grande 
vitesse, sans d'abord penser au développement des trains régionaux " traditionnels " qui rempliraient certainement des missions plus utiles. L'Algérie, par exemple, a passé commande de trains Regiolis en 2015 pour relier Alger, Oran et Constantine à 160 km/ h. Nakamoto et Silveira (2012) font une proposition d'un transport mixte (entre moyenne et grande vitesse) dans le réseau TAV-Brésil, avec l'objectif de promouvoir un développement régional plus efficace, contrairement à « l'effet tunnel » que les trains à grande vitesse ont tendance à générer.

Le processus de prise de décision semble être fortement influencé par des motivations politiques, avec un risque élevé de création d'un projet « too big to fail », dont le coût financier finira par être transféré vers le Trésor Public, et qui pèserait longtemps sur les générations futures.

L'année 2014 a été l'année de la compagne électorale. Il est curieux de penser que très peu a été dit sur le projet TGV-Brésil dans toutes les campagnes des candidats et même en 2015, après la réélection de Dilma Roussef.

\section{BIBLIOGRAPHY}

Ahlfeldt Gabriel et Feddersen Arne, 2010, From periphery to the core: economic adjustments to high speed rail. Disponível em http://www.ieb.ub.edu/aplicacio/fitxers/WS10Ahlfeldt.pdf

Bianca Nakamot et Márcio Silveira, 2012, Projeto TAV Brasil e sistema combinado: grande dilema do planejamento. Revista Formação Online, n. 19, volume 2, p. 132-160.

Beyer Antoine, 2014, La construction d'un espace européen des transports, dossier d'HDR, volume 3, Paris Sorbonne, $318 \mathrm{p}$.

Consórcio Halcrow-Sinergia (2009). Projeto TAV Brasil - Relatório Final. Disponible sur le site www.tavbrasil.gov.br

Junqueira Laurindo, 2012, Trem de alta velocidade : afinal, que trem é esse ? Revista Engenharia, $\mathrm{n}$ 1 , volume 1, p. 100-112.

Mendes Marcos, 2010, Trem de alta velocidade: caso típico de problema de gestão de investimentos.Centro de Estudos da Consultoria do Senado. Texto para Discussão ํo 77.

Mendes Marcos, 2010, Trem de alta velocidade: novas informações para debater o projeto. Centro de Estudos da Consultoria do Senado. Texto para Discussão no 82.

O’Toole, Randal, 2008, High-speed rail: the wrong road for America. Policy Analysis, $\mathrm{n}^{\circ} 625$. CATO Institute. Disponible sur le site http://www.cato.org/pubs/pas/pa-625.pdf.

\section{ABSTRACTS}

The Brazilian government submitted a proposal in 2007 to build the first high-speed railway line to enhance the railway network. Designed to cover $511 \mathrm{~km}$ the railway line will connect three major cities of the south-east region of Brazil: Rio de Janeiro, São Paulo and Campinas. With 
several amendments made to the project scope and timelines, in addition to the lack of companies answering tenders, the project is currently receiving a lot of criticism. The plan is still to relaunch the project in 2014 after the Football World Cup.

En 2007, désireux de rejoindre le contexte de la grande vitesse ferroviaire, le gouvernement brésilien a proposé un projet pour la construction de la première Ligne à Grande Vitesse du pays. Ce projet consiste à parcourir $511 \mathrm{~km}$ en 90 minutes, en connectant trois importantes villes de la région Sud-Est du pays : Rio de Janeiro, São Paulo et Campinas. Après plusieurs rectifications, changements de dates et un manque d'entreprises dans la réponse à d'appel d'offre, le Projet TGV Brésil est actuellement la cible de nombreuses critiques.

Im Jahre 2007 hat die brasilianische Regierung ein Bauprojekt für die erste Hoch Geschwindigkeit Linie des Landes vorgeschlagt. Es handelt sich um die Verknüpfung von drei wichtige Städte des Südöstlichen nationales Raumes : Rio von Janeiro, São Paulo und Campinas. Die Linie würde 511 $\mathrm{Km}$ lang sein und die Reise würde 90 Minuten dauern. Es gab schon mehrere Richtigstellungen und der Kalendär ist verschoben worden. An der öffentliche Auschreibung gaben die vorausgeahnte Unternhemen keine wirkungsvolle Antwort. Jetzt ist das Projekt häufig kritisiert.

\section{INDEX}

Schlüsselwörter: Brasilien, Hoch Geschwindigkeit Linie, Hoch Geschwindigkeit Zug, Projekt Keywords: Brazil, High speed line, high speed train, project

Mots-clés: Brésil, LGV, projet, TGV

\section{AUTHOR}

\section{FRANCISCO CORTEZZI}

Doctorant en Géographie - Université Paris IV - Sorbonne - fmcortezzi@gmail.com 